\title{
URBAN ECOLOGICAL SPACE CHANGES OF 338 PREFECTURE-LEVEL CITIES IN CHINA FROM 2016 TO 2017 WITH HIGH-PRECISION URBAN BOUNDARY AND LAND COVER DATA
}

\author{
Xiaogang Ning ${ }^{1}$, Hao Wang ${ }^{1}$, , Yafei Liu ${ }^{1}$, Minghui Hao ${ }^{1}$, Quan Dong ${ }^{2}$, Wenwen $\mathrm{Xu}^{1}$, Xingfei Cai ${ }^{1}$, Mingzhu Fu ${ }^{1}$, Yinhang Liu ${ }^{1}$, \\ Wenqing Dong 1 \\ ${ }^{1}$ Institute of photogrammetry and remote sensing, Chinese Academy of Surveying and Mapping, Beijing, China - (ningxg, \\ wanghao)@casm.ac.cn \\ ${ }^{2}$ National Quality Inspection and Testing Center for Surveying and Mapping Products, Beijing, China
}

Commission IV, WG IV/3

KEY WORDS: Urban ecological space, National Geoinformation Survey, Land cover change, Spatial analysis, Prefecture-level cities of China

\begin{abstract}
:
Urban ecological space is a significant factor for sustainable urban development and ecological civilization construction. Traditional urban ecological space analysis mainly used medium-resolution image data at large scales and used high-resolution images at a typical urban scale. Few studies focused on the high-precision ecological space analysis at a national urban scale. In this study, highprecision urban boundary and land cover data were utilized to analyze the urban ecological space change and its reason from 2016 to 2017. Ecological space was extracted and merged from high-precision land cover data, which came from the National Geoinformation Survey data of China. Results showed that in 2017, the total urban ecological space area of 338 prefecture-level cities in China was 8514.2 square kilometers, accounting for $22.4 \%$ of the total urban area, which was far below the threshold of $40 \%$ for evaluating the urban green coverage in China. Urban ecological space of 184 cities declined. There were four principal reasons for the decrease of urban ecological space. First, the green space supporting the buildings was occupied. Second, the green landscape space near rivers and lakes was occupied. Third, blocks of woodland were occupied. Fourth, the water area was occupied. The reduced urban ecological space was mainly changed into construction sites, structures, and buildings. Urban ecological space of China was seriously insufficient, and it was heavily occupied. Adequate ecological space should be preserved not only in new urban development areas but also in the old urban areas to ensure people a more comfortable living environment.
\end{abstract}

\section{INTRODUCTION}

Urban ecological space is an indispensable urban space that guarantees urban ecological security and improves the quality of life of residents. Ecological space is also known as green space or ecological land (Wang et al., 2017). The Main Function Area Planning of China mentioned that the current national land space development faced with the problems of more space occupied by industrial and mining construction and a sharp reduction in green ecological space. Strategy of Ecological Civilization Construction of China pointed out that it was necessary to build a balanced and suitable urban and rural construction space system, appropriately increase living space and ecological land, and protect and expand green spaces, water areas, wetlands and other ecological spaces. The report of the Eighteenth National Congress of the Communist Party of China proposed to build an ecological civilization to achieve intensive and efficient production space, moderate and livable living space, and picturesque ecological space. The National New Urbanization Plan of China (2014-2020) stated that it was necessary to reasonably delimitate the ecological protection red line, expand the urban ecological space, and build green ecological corridors in urbanized areas. Ecological space control has become an essential part of land space control in China. As the impact of global climate change continues to intensify, the protection and expansion of green ecological spaces face more severe challenges.

Remote sensing technology provides an essential means for monitoring the area and pattern of ecological space. However, the current urban ecological space monitoring research is mainly limited to low- and medium-resolution images (Kuang, 2019; Rafiee et al., 2009), while high-resolution images are mostly limited to a specific area (Haas and Ban, 2017; Huang et al., 2004). There is little research on the high-precision urban ecological space at the national or large regional scale. Besides, as the basic unit of urban ecological space analysis, the urban boundary lacks accurate products in large-scale. The large-scale urban boundary is mainly obtained from low- and mediumresolution images (Gong et al., 2020; Imhoff et al., 1997), while the urban boundary lacks spatial details with poor product accuracy and cannot fully reflect the characteristics of urbanrural development differences (Wang et al., 2012; Zhang et al., 2018). High-resolution images provide the potential for highprecision urban boundary extraction. However, the existing studies mostly focused on the scale of a single city or a small area, and the separation of urban and rural areas is less considered. Urban boundaries are generally expressed in terms of residential areas and buildings (Ning and Lin, 2017; Tao et al., 2010), which cannot meet the needs of refined management.

* Corresponding author 
Some have integrated high-resolution images better than 2 meters and separation characteristics into the extraction of urban boundaries, providing opportunities for high-precision analysis of urban spatial patterns (Ning et al., 2018; Wang et al., 2018).

To solve the above problems, this study uses high-precision land coverage data and high-precision urban boundary data obtained with a resolution of better than 2 meters to carry out ecological space delineation within the urban areas. Three hundred thirty-eight prefecture-level cities across the country are taken as research areas to analyze the urban ecological space changes from 2016 to 2017, to provide recommendations for the national urban ecological space risk assessment and how urban residents can get a more comfortable life.

\section{MATERIALS AND METHODS}

\subsection{Study Area and Data}

Three hundred thirty-eight prefecture-level cities of 31 provincial administrative units, excluding Hong Kong, Macao, and Taiwan, were selected as study areas. Since the prefecturelevel cities are the economic and population centers of the provincial administrative units, they can account for the highest level of urbanization in the province. A study on urban ecological space of prefecture-level cities will help people understand the overall urbanization of China.

High-precision land coverage data were from the National Geoinformation Survey Data of China and updated annually. Data production, quality inspection, and acceptance are organized by the Ministry of Natural Resources of the People's Republic of China. The resolution of the images used for land coverage data production is better than 2 meters, and the quality is guaranteed by manual interpretation and field verification. The accuracies of the positioning points and the classification boundary, which is clear in the images, are better than 5 pixels. The data have the advantages of high accuracy, rich classification content, substantial presence, and seamless ground coverage, which reflects the natural attributes or conditions of natural and artificial structures on the surface (Ministry of Natural Resources, 2019). It adopts a three-level classification system. There are 10 first-level categories, 52 second-level categories, and 104 third-level categories. Firstlevel categories include cultivated land, forest and grass cover, buildings, traffic networks, structures, construction sites, deserts and bare lands, and waters.

High-resolution remote sensing images were collected by the Ministry of Natural Resources of the People's Republic of China, and the resolution is better than 2 meters. The images were projected onto the CGCS2000 coordinate system after geometric correction. Geographic boundaries from National Geoinformation Survey Data of China, which includes urban roads, place name address data, township, and county-level administrative divisions, were also collected. Geographic boundaries can be used for high-precision city boundary extraction.

\subsection{Urban Boundary and Ecological Space Extraction}

Considering the urban landscape characteristics, continuous patches and the regional development differences, urban boundaries were extracted by using high-resolution remote sensing images as the primary data source, combined with thematic information such as urban roads, place names and administrative divisions (Ning et al., 2018; Wang et al., 2018).
Urban boundary extraction followed six geographic rules, which are the limitation of administrative division, the drawing of urban boundary outline, the decision of central centralized and adjacent region, the decision of central centralized and adjacent region, the judgment of urban landscape, the judgment of enclave urban areas, and the inverse extraction of urban boundary from new time to old time. Compared with MODIS and Landsat urban boundary data products, the urban boundary data with this method can avoid the misclassification and omission of urban boundaries and can distinguish urban areas from the urban-rural fringe and rural construction land (Wang et al., 2018; Zhang et al., 2018). Regarding the results of previous studies (Wang et al., 2017), two first-level categories, which are forest and grass cover, water, were selected from the land cover data of National Geoinformation Survey Data of China and combined to represent ecological space. Urban boundaries were used to cut the ecological space to obtain the urban ecological space.

\subsection{Spatial Statistic Method}

The area of urban ecological space, its proportion to the urban extent, and the change of the above two indicators from 2016 to 2017 were used as the statistical analysis indicators for 338 prefecture-level cities. $40 \%$ was used as the threshold for evaluating whether the proportion of urban ecological space was good or not according to "The greening coverage of urban planning and construction areas across the country should be over $40 \%$ by 2010 " proposed in Notice of the State Council on Strengthening Urban Greening Construction of China. The reasons for the change of urban ecological space were analyzed from two aspects. The transformation of land cover types from the reduced urban ecological space was analyzed by using the land cover data. Besides, the main reasons for the reduced urban ecological space were explained by manual visual interpretation and judgment based on high-resolution images.

\section{RESULT AND DISCUSSION}

\subsection{Area of Urban Ecological Space and The Proportion}

In 2017, the total urban ecological space area of 338 prefecturelevel cities of China was 8514.2 square kilometers, accounting for $22.4 \%$ of the total area of urban areas. The median value of urban ecological space proportion was $19.35 \%$. Both the mean value and the median value were far below $40 \%$, indicating a severe shortage of urban ecological space. Only 100 cities arrived at the mean value of $22.4 \%$ of urban ecological space proportion. There were only 12 cities with more than $30 \%$ urban ecological space, namely Yanji, Daqing, Sanya, Suzhou, Karamay, Binzhou, Zhongwei, Changji, Huanggang, Dongying, Haikou, and Yingtan. Yanji had the most significant proportion of urban ecological space, which was just 39\%. Even 15 cities had a proportion of urban ecological space of less than $10 \%$. Although the proportion of urban ecological space of 338 prefecture-level cities increased by $0.23 \%$ from 2016 to 2017 at the national scale, that in more than half (184) cities decreased at the single city scale. The situation of Urban ecological space protection was grim. Decrease of proportion of urban ecological space mainly occurred in Guangdong, Xinjiang, Anhui, Guangxi, Henan, Hubei, Liaoning, Sichuan, Shanghai, Tianjin, and Chongqing (Figure 1). Among these provinces, the number of cities with a decreased proportion of urban ecological space in Guangdong, Xinjiang, Anhui, Guangxi, Henan, Hubei, Liaoning, Sichuan arrived at 10 . The highest number of cities in Guangdong was 14. 


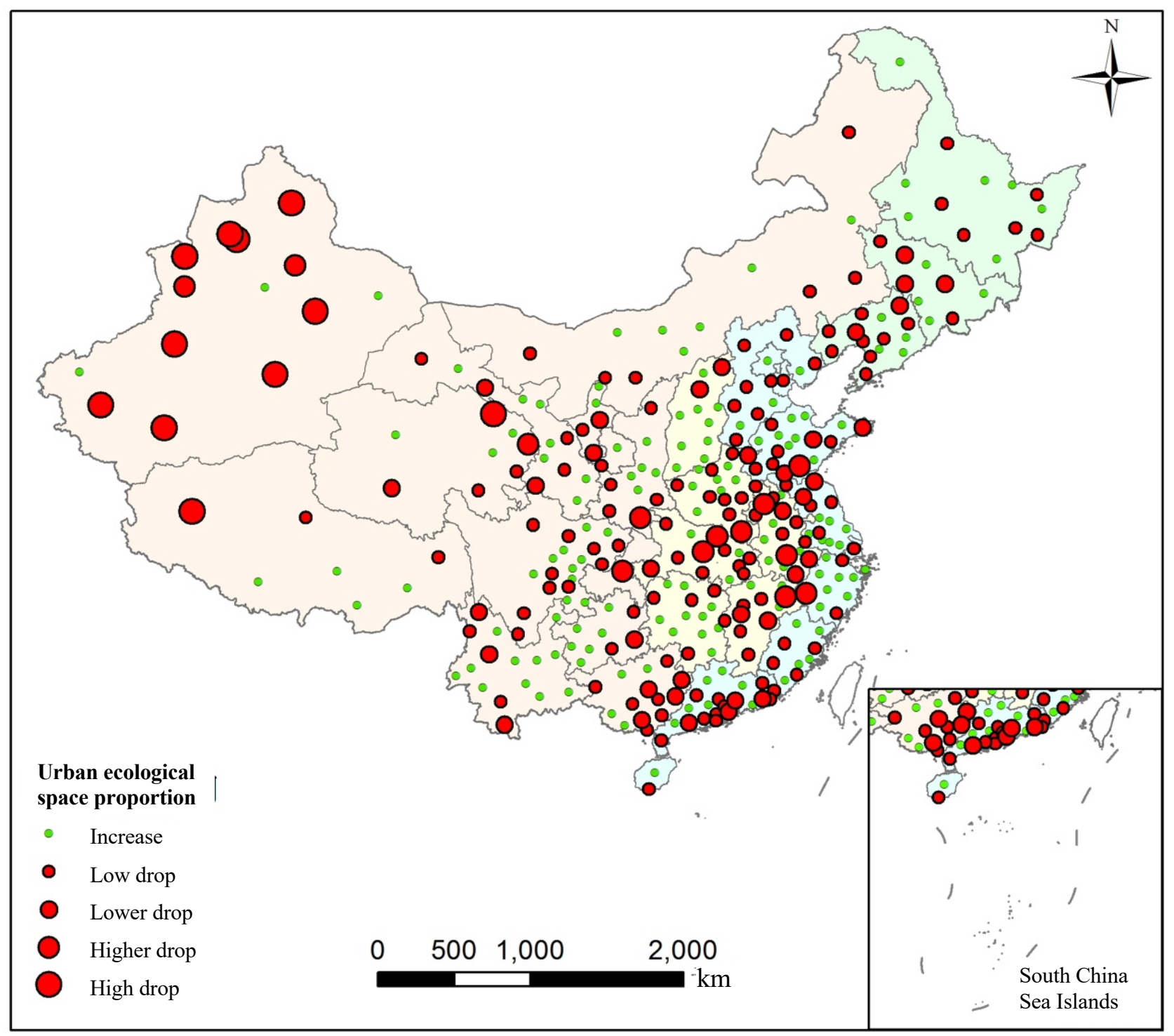

Figure 1. Hierarchical map of urban ecological space proportion change of 338 prefecture-level cities in China from 2016 to 2017

\subsection{Reasons of Urban Ecological Space Change}

Fifty-seven cities with a significant decrease in urban ecological space were taken as the sample cities for analyzing the reasons for urban ecological space change. Generally, the reduced ecological space mainly transformed into three types of land cover, which were construction sites, structures, and buildings (Figure 2). Among them, 57.9\% of cities have the largest proportion of urban ecological space transformed to construction sites, followed by structures, $31.6 \%$; buildings accounted for $5.3 \%$. $19.3 \%$ of cities had the proportion of urban ecological space exceeds 50\%, which transformed to construction sites. With the help of high-resolution remote sensing images, results showed that there were four principal reasons for the decrease of urban ecological space. First, the green space supporting the buildings was occupied (Figure 2a). Second, the green landscape space near rivers and lakes was occupied (Figure $2 b$ ). Third, blocks of woodland in urban areas were occupied (Figure 2c). Fourth, the water area in the urban areas was occupied (Figure 2d).
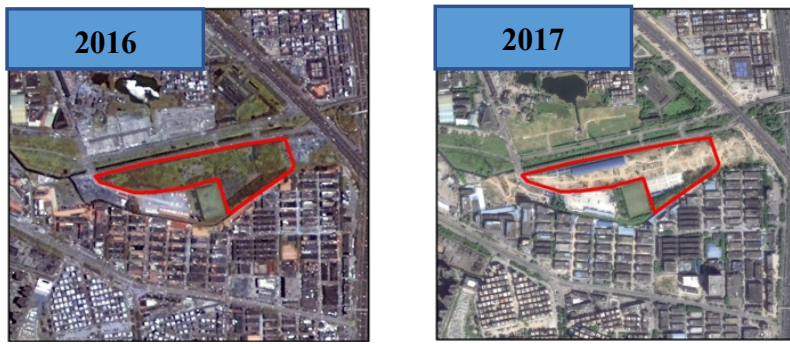

a. Green space supporting the buildings was occupied
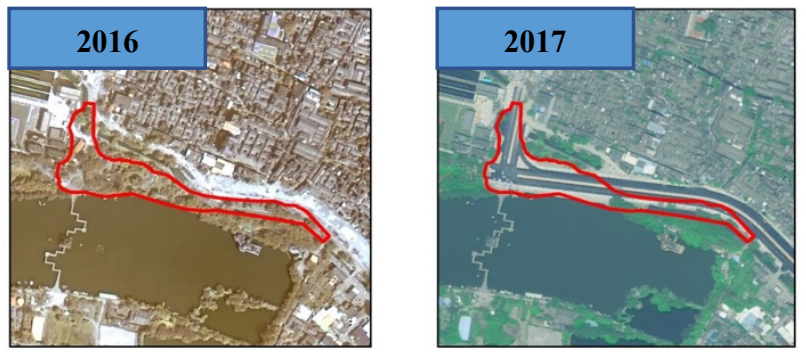

b. Green landscape space near the river and lake was occupied 

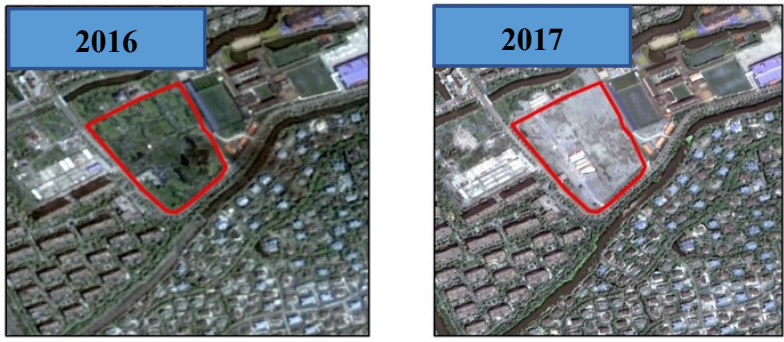

c. Blocks of woodland in urban area was occupied
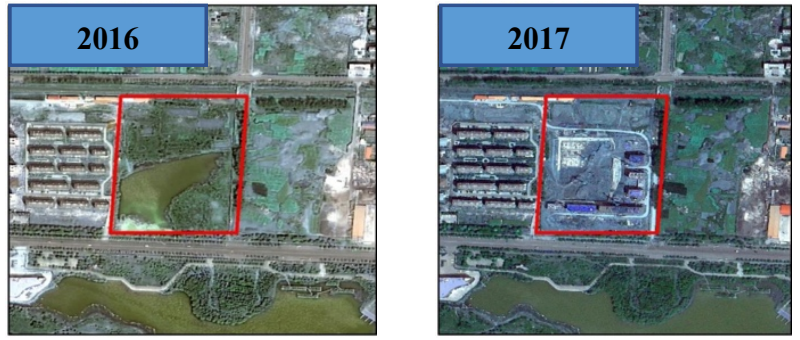

d. Water area in urban area was occupied

Figure 2. Schematic diagram of occupied ecological space from 2016 to 2017

\section{CONCLUSION}

In 2017, the area of urban ecological space of 338 prefecturelevel cities in China was only $22.4 \%$ of the total area of urban areas, and more than half of the cities had a decreased urban ecological space proportion. The reduction of green space supporting the buildings, green landscape space near rivers and lakes, blocks of woodland, and water area were the major reasons for the reduction of urban ecological space. In the process of construction and development in most cities, emphasizing economic benefits over ecological and environmental benefits still exists. The reduction of urban ecological space is prominent, and the situation of urban ecological construction and protection is severe, so it is urgent to carry out special monitoring and evaluation projects at national and city levels.

\section{ACKNOWLEDGEMENTS}

This research is supported by National Natural Science Foundation of China (NO: 41401513) and the National Geoinformation Survey Project by the Ministry of Natural Resources of the People's Republic of China.

\section{REFERENCES}

Gong, P., Chen, B., Li, X., Liu, H., Wang, J., Bai, Y., Chen, J., Chen, X., Fang, L., Feng, S., 2020. Mapping essential urban land use categories in china (EULUC-China): Preliminary results for 2018. Science Bulletin, 65(3), 182-187.

Haas, J., Ban, Y.F., 2017. Mapping and monitoring urban ecosystem services using multitemporal high-resolution satellite data. IEEE Journal of Selected Topics in Applied Earth Observations and Remote Sensing, 10(2), 669-680.

Huang, H., Wu, B., Li, M., Zhou, W., Wang, Z., 2004. Detecting urban vegetation efficiently with high resolution remote rensing data. Journal of Remote Sensing, 8(1), 68-74.
Imhoff, M.L., Lawrence, W.T., Stutzer, D.C., Elvidge, C.D., 1997. A technique for using composite dmsp/ols "city lights" satellite data to map urban area. Remote Sensing of Environment, 61(3), 361-370.

Kuang, W., 2019. Mapping global impervious surface area and green space within urban environments. Science China Earth Sciences, 49(7), 1151-1168.

Ministry of Natural Resources of the People's Republic of China, 2019. Content and index of fundamental geographic conditions monitoring, China.

Ning, X., Lin, X., 2017. An index based on joint density of corners and line segments for built-up area detection from high resolution satellite imagery. ISPRS International Journal of Geo-Information, 6(11), 338.

Ning, X., Wang, H., Lin, X., Cao, Y., Du, J., 2018. Spatiotemporal urban sprawl monitoring and analysis over beijingtianjin-hebei urban agglomeration during 1990-2015. Acta Geodaetica Et Cartographica Sinica, 47(9), 1207-1215.

Rafiee, R., Mahiny, A.S., Khorasani, N., 2009. Assessment of changes in urban green spaces of mashad city using satellite data. International Journal of Applied Earth Observation and Geoinformation, 11(6), 431-438.

Tao, C., Tan, Y., Cai, H., Bo, D.U., Tian, J., 2010. Objectoriented method of hierarchical urban building extraction from high-resolution remote-sensing imagery. Acta Geodaetica Et Cartographica Sinica, 39(1), 39-45.

Wang, F., Wang, K., Chen, T., Li, P., 2017. Progress and prospect of research on urban ecological space. Progress in Geography, 36(2), 207-218.

Wang, H., Ning, X., Zhang, H., Liu, Y., Yu, F., 2018. Urban boundary extraction and urban sprawl measurement using highresolution remote sensing images: A case study of china's provincial capital. International Archives of the Photogrammetry, Remote Sensing and Spatial Information Sciences, XLII-3, 1713-1719.

Wang, L., li, C., Ying, Q., Cheng, X., Wang, X., Li, X., Hu, L., Liang, L., Yu, L., Huang, H., Gong, P., 2012. China's urban expansion from 1990 to 2010 determined with satellite remote sensing. Chinese Science Bulletin, 57(22), 2802-2812.

Zhang, H., Ning, X., Wang, H., Shao, Z., 2018. High accuracy urban expansion monitoring and analysis of china's provincial capitals from 2000 to 2015 based on high-resolution remote sensing imagery. Acta Geographica Sinica, 73(12), 2345-2363. 